Annals of Pure and Applied Mathematics

Vol. 21, No. 2, 2020, 141-147

ISSN: 2279-087X (P), 2279-0888(online)

Published on 16 June 2020

www.researchmathsci.org

DOI: http://dx.doi.org/10.22457/apam.v21n2a10642

$\overline{\text { Annals of }}$

Pure and Applied

Mathematics

\title{
The Classification of Some Order Four Digraphs As Nonnegative $\mathbf{P}_{\mathbf{0 , 1}}$-Matrix Completion
}

\author{
Agnes Ngima Ngetha ${ }^{1}$ and Victor Kemboi Tomno ${ }^{2 *}$ \\ ${ }^{1}$ Department of Mathematics and Physical Science \\ Dedan Kimathi University of Technology \\ Private Bag -10143, Dedan Kimathi, Kenya \\ ${ }^{2}$ Department of Mathematics Physics, Moi University \\ P.O Box 3900-30100, Eldoret, Kenya \\ ${ }^{1}$ E-mail: ngethaagnes@gmail.com \\ *Corresponding author. ${ }^{2}$ E-mail: victomno@gmail.com
}

Received 31 March 2020; accepted 12 June 2020

Abstract. A digraph of order $n$ is said to have nonnegative $P_{0,1}$-completion if every partial nonnegative $P_{0,1}$-matrix that specifies the digraph can be completed to a nonnegative $P_{0,1}$ matrix. The aim of this paper is to classify some digraphs of order four that include all loops as either having nonnegative $P_{0,1}$-completion or not having nonnegative $P_{0,1}$ completion.

Keywords: Matrix completion, digraphs, partial matrix, nonnegative $P_{0,1}$-matrix, nonnegative $P_{0,1}$-completion

AMS Mathematics Subject Classification (2010): 15A48, 05C50

1. Introduction

In this section, we recall and introduce some of the definitions and fundamental concepts that play a key role in this paper.

Definition 1.1. A real $n \times n$ matrix A is a $P$ (resp. $P_{0}$ )-matrix if all principal minor of the matrix $A$ are positive (resp. nonnegative). A $P_{0}$-matrix A with all its diagonal entries being positive is called a $P_{0,1}$-matrix.

Definition 1.2. A nonnegative $P\left(\right.$ resp. $\left.P_{0}, P_{0,1}\right)$-matrix is a $P\left(\right.$ resp. $\left.P_{0}, P_{0,1}\right)$-matrix in which all entries are nonnegative. 


\section{Agnes Ngima Ngetha and Victor Kemboi Tomno}

Definition 1.3. A partial matrix is a matrix in which some entries are specified and others are not specified.

Let $\pi$ be $P, P_{0}, P_{0,1}$, nonnegative $P$, nonnegative $P_{0}$ or nonnegative $P_{0,1}$ then the following definitions.

Definition 1.4. A partial $\pi$-matrix is a partial matrix in which all specified entries satisfies the conditions of a $\pi$-matrix.

Definition 1.5. A completion of a partial matrix is a specific choice of values for the unspecified entries resulting in a required matrix.

During the completion of partial matrices, digraphs are used and in some papers they use patterns which means same thing in matrix completions. Therefore, we have the following useful definitions for patterns and digraphs.

Definition 1.6. A pattern $Q$ for $n \times n$ matrices is a list of positions of the specified entries of a matrix. In view of a digraphs, a digraph of a pattern for $n \times n$ matrix is the digraph $D=\left(V_{D}, A_{D}\right)$ with set $V_{D}$ of vertices equal to $\{1, \ldots, n\}$ and set $A_{D}$ of arcs equal to the ordered $(i, j)$ such that $i \neq j$ and position $(i, j)$ is in the pattern or in other words, if an arc exist between vertices $i$ and $j$ then we say position $(i, j)$ of the partial matrix is specified.

Definition 1.7. The order of a digraph $D$ denoted $n$ is the number of vertices of $D$. A digraph is complete digraph or clique if it includes all possible arcs between its vertices, and if patterns then all positions $(i, j)$ are listed. A digraph is called symmetric if an arc $(i, j)$ exist if and only if $(j, i)$ is in the graph and is called asymmetric if an $\operatorname{arc}(j, i)$ is in the graph then $(j, i)$ does not exist in the graph. A path in a digraph is a sequence of vertices $v_{1}, \ldots, v_{k}$ in $V_{D}$ such that for $i=1, \ldots, k-1$, the arc $\left(v_{i}, v_{i+1}\right) \in A_{D}$ and all vertices are unique except maybe $v_{1}=v_{k}$ and if that is the case then it is called $k$-cyclic digraph [9].

Definition 1.8. A partial matrix $A$ is said to specify a pattern-digraph if its specified entries lie exactly in those positions listed in the pattern or for a digraph position $a_{i j}$ of the partial matrix $A$ is specified if and only if there is an arc between vertex $v_{i}$ and $v_{j}$ of $D$.

Example 1.9. Consider the digraph in Figure 1.

The digraph in Figure 1, is of order 3 with 4 arcs (non-loops), and it is 3-cycle with the path $v_{1} \rightarrow v_{2} \rightarrow v_{3} \rightarrow v_{1}$. 
The Classification of Some Order Four Digraphs as Nonnegative $\mathrm{P}_{0,1}$-Matrix Completion

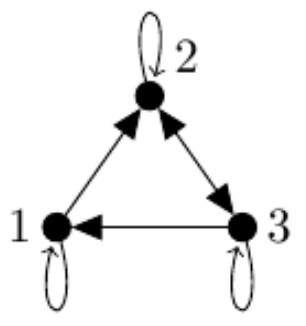

Figure 1: Digraph of order 3

The partial matrix that specifies digraph in Figure 1 is given below.

$$
\left[\begin{array}{ccc}
d_{1} & a_{12} & x_{13} \\
x_{21} & d_{2} & a_{23} \\
a_{31} & a_{32} & d_{3}
\end{array}\right]
$$

In the partial matrix $d_{i}$ denoted the specified diagonal entries, in the digraph is the loops. $a_{i j}$ is the specified entry and in the digraph it represents the arcs (non-loops) and finally, $x_{i j}$ is the unspecified non-diagonal entries and in the digraphs it represents those vertices that do not have arcs between them.

Translating above to a pattern we have, $Q=\{(1,1),(1,2),(2,2),(2,3),(3,1),(3,2),(3,3)\}$

Definition 1.10. A pattern (or its (di)graph) has $\pi$-completion if every partial $\pi$ matrix which specifies the pattern (or its (di)graph) can be completed to a $\pi$-matrix. Note that if a pattern contains all the diagonal entries then the digraph specified contains loops in all vertices.

All non-isomorphic digraphs of order at most four are given in [9], where $p$ denotes number vertices, $q$ denotes the number of arcs and for non-isomorphic digraphs with equal number of vertices and arcs, it has a position number denoted $n$. Whenever a digraph has only one non-isomorphic digraph then there is no need of using $n$ the digraph number.

In Section 2, we discuss closely related work leading to this problem and in Section 3 , we give preliminary results in this paper. The main results on classification of digraphs of order 4 regarding nonnegative $P_{0,1}$-completion are given in Section 4 .

\section{Related work}

Over the past years, several matrix completion problems have been considered. The authors in [1] showed that every symmetric partial $P$-matrix has $P$-completion. Another paper on same class [2] proved that any pattern of $3 \times 3$ matrices has $P$ completion. Furthermore, they classified 207 out of 218 digraphs as either having $P$ completion or not having $P$-completion. Hogben [3] proved that every digraph of order 
Agnes Ngima Ngetha and Victor Kemboi Tomno

3 has nonnegative $P$-completion and in [4] all digraphs of order 4 were classified regarding nonnegative $P$-completion.

Another related class is of $P_{0}$-matrices and its subclass nonnegative $P_{0}$-matrices. It was noted in Example 9.1 of [3] and Example 3.1 of [5] that not all digraphs of order 3 have $P_{0}$-completion. Moreover, authors in [5] successfully classified all digraphs of order 4 as either having $P_{0}$-completion or not having $P_{0}$-completion. A year later, it was shown in [6] that all digraphs of order 3 that includes all loops have nonnegative $P_{0}$ completion. In addition, they provided complete classifications of digraphs of order 4 as nonnegative $P_{0}$-completion.

The third and closely related class is of $P_{0,1}$-matrices. In 2001, Hogben [3] proved that all digraphs of order 3 have both $P_{0,1}$-completion and nonnegative $P_{0,1}$ completion. Wangsness [7] in her $\mathrm{PhD}$ thesis extended the work of Hogben [3], she classified 192 out of 218 digraphs of order 4 regarding $P_{0,1}$-completion. In the present paper, we will classify some digraphs of order 4 as having nonnegative $P_{0,1}$-completion or not having $P_{0,1}$-completion.

\section{Preliminary results}

In this section, we will present some basic results that will be useful in the next section when classifying digraphs of order four. We first present classifications of digraphs order 2 and 3 in Theorem 3.1 and Theorem 3.2 respectively.

Theorem 3.1. [3, Lemma 4.8] All patterns for $2 \times 2$ matrices have nonnegative $P_{0,1}$ completion.

Theorem 3.2: [3, Lemma 8.1] A pattern for $3 \times 3$ matrices with all specified diagonal entries has nonnegative $P_{0,1}$-completion.

According to [3, Example 4.3], it was clear that some partial nonnegative $P_{0,1}$ matrices with some unspecified diagonal entries cannot be completed to a nonnegative $P_{0,1}$-matrices. This leads us to some necessary conditions of nonnegative $P_{0,1}$-matrices of having all diagonal entries specified or rather we use digraphs that include all loops.

Lemma 3.3. [3, Theorem 8.4] Any symmetric $n$-cycle that has loops at every vertex has nonnegative $P_{0,1}$-completion.

Lemma 3.4. [8, Theorem 2.13] Any asymmetric pattern that has non-negative $P$ completion also has nonnegative $P_{0,1}$-completion. 
The Classification of Some Order Four Digraphs as Nonnegative $\mathrm{P}_{0,1}$-Matrix Completion Lemma 3.5. [8, Theorem 2.12] Any pattern that has nonnegative $P_{0}$-completion also has nonnegative $P_{0,1}$-completion.

Lemma 3.6. [8, Corollary 2.9] Any pattern that has nonnegative $P_{0,1}$-completion also has nonnegative $P$-completion.

We will use Lemmas 3.3, 3.4 and 3.5 to list some digraphs of order 4 having nonnegative $P_{0,1}$-completion and Lemma 3.6 will be used to list some digraphs that do not have nonnegative $P_{0,1}$-completion.

\section{Main results}

We are now able to classify most of digraphs of order 4 as having nonnegative $P_{0,1}$ completion or not having nonnegative $P_{0,1}$-completion.

Theorem 4.1: The following digraphs of order 4 have nonnegative $P_{0,1}$-completion

$$
\begin{array}{lll}
q=0 ; & & \\
q=1 ; & & \\
q=2, & & n=1-5 ; \\
q=3, & & n=1-13 ; \\
q=4 & n=1-27 \\
q=5 & n=1-33,35-38 ; \\
q=6 & n=1-21,23-32,35,36,38-41,43,44,46-48 ; \\
q=7 & n=1-7,13-17,19,21-24,26,28,29,31,34,36,37 ; \\
q=8 & n=1-2,6,10,12,14,15,18,21,27 \\
q=9 & n=8,11 ; \\
q=12 & &
\end{array}
$$

\section{Proof:}

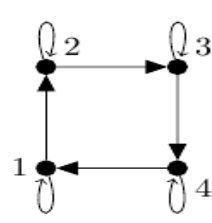

$q=4, n=16$

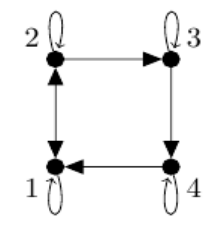

$q=5, n=7$

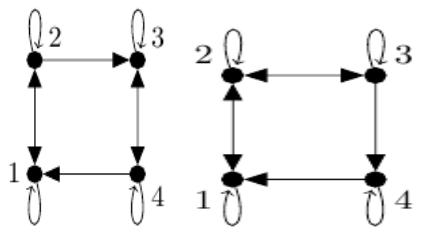

$q=6, n=4$

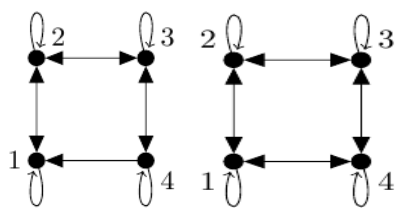

$q=7, n=2 \quad q=8, n=2$ 
Agnes Ngima Ngetha and Victor Kemboi Tomno

Digraph $q=8 n=2$ is a symmetric 4-cycle and in view of Lemma 3.3, it has nonnegative $P_{0,1}$-completion.

A partial nonnegative $P_{0,1}$-matrix specifying any of the digraphs $q=4 n=16$; $q=5 n=7 ; q=6 n=4,7$ and $q=7 n=2$ may be extended to a partial nonnegative $P_{0,1}$-matrix specifying $q=8 n=2$ by setting all unspecified entries to zeros. Therefore, all these digraphs have nonnegative $P_{0,1}$-completion.

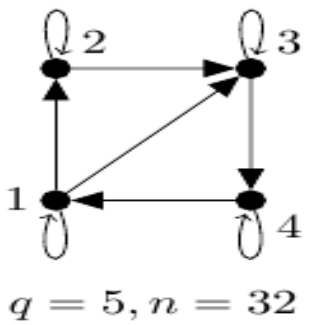

Digraph $q=5 n=32$ is an asymmetric digraph and it has nonnegative $P$-completion by Theorem 2.6 of [5]. Thus it also has nonnegative $P_{0,1}$-completion by Lemma 3.4.

According to [6, Lemmas 2.2, 2.3, 2.4 and 2.5], all the remaining digraphs listed above have nonnegative $P_{0}$-completion. By Lemma 3.5, they also have nonnegative $P_{0,1}$ -completion.

Observe also that digraph $q=4 n=16$ is asymmetric and has nonnegative $P$ completion then one can still use Lemma 3.4 to proof that it has nonnegative $P_{0,1}$ completion.

Theorem 4.2. The following digraphs of order 4 do not have nonnegative $P_{0,1}$ completion

$$
\begin{aligned}
& q=6 \quad n=45 \text {; } \\
& q=7 \quad n=30,32,33,35,38 \text {; } \\
& q=8 \quad n=16,17,19,20,22-27 \text {; } \\
& q=9 \quad n=3-7,9,10,12,13 \text {; } \\
& q=10 \quad n=1-5 \text {; } \\
& q=11 \text {. }
\end{aligned}
$$

Proof: According to [4, Theorem 2.6], each of the digraphs listed do not have nonnegative $P$-completion. Hence, by Lemma 3.6 these digraphs do not have nonnegative $P_{0,1}$-completion.

Remark 4.3. The main focus of this paper was to classify digraphs of order four as nonnegative $P_{0,1}$-completion. However, the following 23 digraphs were not classified 
The Classification of Some Order Four Digraphs as Nonnegative $\mathrm{P}_{0,1}$-Matrix Completion and one may study their completeability and classify them as having nonnegative $P_{0,1}$ completion or not having nonnegative $P_{0,1}$-completion.

$$
\begin{array}{ll}
q=6 & n=22,33,34,37,42 ; \\
q=7 & n=8,10,12,13,18,20,25,27 ; \\
q=8 & n=3-5,7-9,11,13 ; \\
q=9 & n=1-2 ;
\end{array}
$$

\section{REFERENCES}

1. C.R.Johnson and B.K.Kroschel, The combinatorially symmetric $P$-matrix Completion Problem, Electronic Journal of Linear Algebra, 1 (1996) 59-64.

2. L.M.DeAlba, and L.Hogben, The completions $P$-matrix patterns, Linear Algebra and Application, 319 (2000) 83-102.

3. L.Hogben, Graph Theoretic Methods of Matrix Completion Problem, Linear Algebra and its Applications, 328 (2001) 161-202.

4. J.Bowers, J.Evers, L.Hogben, S.Shaner, K.Snider and A.Wangsness, On completion problems for various classes of $P$-matrices, Linear Algebra and Application, 413 (2006) 342-354.

5. J.Y.Choi, L.M.Dealba, L Hogben and M.Maxwell, The $P_{0}$-Matrix Completion Problem, Electronic Journal of Linear Algebra, 9 (2002) 1-20.

6. J.Y.Choi, L.M.DeAlba, L.Hogben, B.M.Kivunge, S.K.Nordstrom and M.Shedenhelm, The nonnegative $P_{0}$-matrix completion problem, Electronic Journal of Linear Algebra, 10 (2003) 46-59.

7. A.Wangsness, The matrix completion problem regarding various classes of $P_{0,1}$ matrices, PhD Thesis, Iowa State University, 2005.

8. L.Hogben, Matrix completion problems for pairs of related classes of matrices, Linear Algebra and its Applications, 373 (2003) 13-29

9. F.Harary, Graph Theory; New York; Addison-Wesley Publishing Company, 1969. 